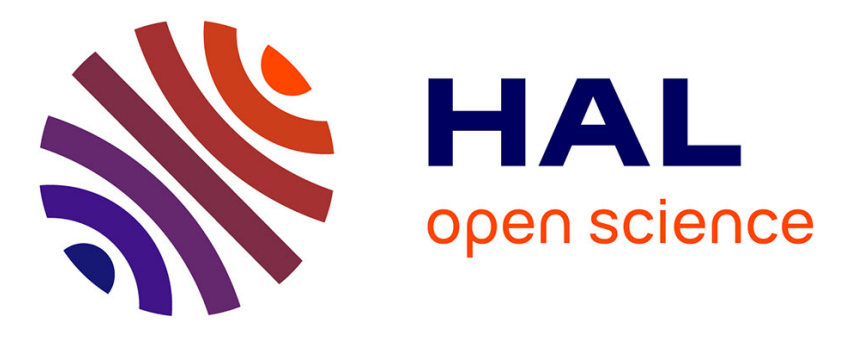

\title{
La nomination des métiers dans l'œuvre de Diego Sánchez de Badajoz (théâtre espagnol du XVIe siècle) \\ Françoise Cazal
}

\section{To cite this version:}

Françoise Cazal. La nomination des métiers dans l'œuvre de Diego Sánchez de Badajoz (théâtre espagnol du XVIe siècle). Georges Hanne, Claire Judde de Larivière. Noms de métiers et catégories professionnelles. Acteurs, pratiques, discours (XVe siècle à nos jours), Framespa/Université de Toulouse-Le Mirail, pp.51-67, 2010, Méridiennes. halshs-00386095

\section{HAL Id: halshs-00386095 https://shs.hal.science/halshs-00386095}

Submitted on 20 Nov 2009

HAL is a multi-disciplinary open access archive for the deposit and dissemination of scientific research documents, whether they are published or not. The documents may come from teaching and research institutions in France or abroad, or from public or private research centers.
L'archive ouverte pluridisciplinaire HAL, est destinée au dépôt et à la diffusion de documents scientifiques de niveau recherche, publiés ou non, émanant des établissements d'enseignement et de recherche français ou étrangers, des laboratoires publics ou privés. 


\title{
La nomination des métiers dans l'œuvre de Diego Sánchez de Badajoz (théâtre espagnol du XVI ${ }^{e}$ siècle)
}

\author{
Françoise Cazal \\ Université de Toulouse \\ FRAMESPA-UMR 5136 CNRS \\ LEMSO
}

Le rôle joué par les corporations dans le financement des représentations théâtrales de la Fête-Dieu en Espagne, au XVI siècle, n'implique pas forcément la mention des métiers commanditaires dans les œuvres elles-mêmes. Ces métiers sont pourtant bien présents dans le thêâtre religieux de Diego Sánchez de Badajoz, en Estrémadure, un théâtre qui baigne dans une atmosphère pré-tridentine et où se combinent intentions didactiques et comiques. Certes, nous sommes bien loin des listes d'enregistrement, objet d'étude des historiens dans le présent colloque ; mais, dans le cadre de ces textes littéraires, aussi, se produit une nomination insistante des métiers, ou plus exactement des " professions ", l'emploi de ce terme étant justifié, puisque nous verrons qu'il y a revendication de compétences, intention déclarative et mention systématisante, et surtout, au plus haut degré, valeur expressive, puisqu'il s'agit de théâtre. Nous ne tombons pas dans l'illusion qui consisterait à voir dans un texte dramatique une représentation exacte de la réalité historique; mais les modes de pensée et d'expression observables dans ces textes s'inscrivent tout de même légitimement dans le vaste volet de l'histoire culturelle du social : le théâtre est, lui aussi, indirectement, un dispositif de connaissance des populations, si l'on garde à l'esprit que les enjeux attachés à telle ou telle profession et les positions hiérarchiques qui y sont liées apparaissent traduits de façon spécifique dans ce langage particulier qu'est le langage dramatique.

Nous nous attacherons ici à montrer comment sont mises en théâtre les professions de pêcheur, de maréchal-ferrant, de forgeron, de meunier, d'apiculteur et de jardinier, dans ces Farsas écrites entre 1530 et 1552 par Diego Sánchez de Badajoz, curé d'un bourg proche de cette ville, Talavera la Real, et publiées en 1554 par le neveu du dramaturge, peu après la mort de l'auteur. Sur la personne même du dramaturge, on ne sait presque rien, si ce n'est qu'il avait le plus modeste grade universitaire, celui de Bachiller, et qu'il a signé pendant vingt-cinq ans le registre des naissances de sa paroisse. La notoriété locale de ce dramaturge était importante et ses pièces étaient représentées à la capitale, Badajoz, et jouées dans le palais des Feria, dont il recevait quelque soutien, si l'on en juge par les travaux d'Ann Wiltrout ${ }^{1}$. Ce théâtre est nettement ancré dans un univers populaire et son personnage central, le Berger, bien qu'étant une figure littéraire héritée d'un autre dramaturge, Torres Naharro, est nourri de verve rustique authentique.

Les vingt-huit pièces de ce dramaturge sont réunies dans un recueil intitulé Recopilación en metro (' Collection de textes en vers ') ${ }^{2}$. Ces

1 A. Wiltrout, A Patron and a Playwright in Renaissance Spain : The House of Feria and Diego Sánchez de Badajoz, London, Tamesis, 1987.

2 D. Sánchez de Badajoz, Recopilación en metro, Sevilla, 1554, F. Weber de Kurlat, "Seminario Dr. Amado Alonso ", Universidad de Buenos Aires, Buenos Aires, 1968. Textes étudiés :

- Yntroito de pescadores (' Prologue des pêcheurs ') de tierra de Badajoz, p. 553-556.

- Yntroito de herradores (' Prologue des maréchaux-ferrants '), p. 561-563

- Farsa del herrero ('Farce du forgeron') en que entran tres figuras : un Herrero martillando junto a su fragua, $y$ un Pastor $y$ un romero, p. 469-473. 
pièces, de longueur très variable (de 120 à 1200 vers), sont conçues pour célébrer les grandes dates du calendrier religieux ${ }^{3}$, Noël, pâques ou la fête d'un saint, mais surtout la Fête-Dieu, comme c'est le cas des pièces qui nous intéressent ici. Dans le cadre de cette fête, les corporations et confréries rivalisent pour honorer le saint-Sacrement en participant aux processions et en étant les commanditaires de pièces de théâtre destinées à être jouées dans la rue ou sur le parvis de l'église. Ces Farsas où la composante comique, destinée à mieux faire passer le message religieux didactique, est très développée, comportent généralement trois parties, un prologue appelé introito, suivi du corps de la farce et d'un final chanté. Le prologue est traditionnellement récité par un personnage bien à part, le Berger, issu du théâtre religieux primitif et chargé de connotations folkloriques populaires, personnage qui, parmi ses multiples fonctions, a celle de présenter la pièce au public. Les six professions étudiées ici sont dramatisées dans quatre pièces complètes et dans deux introitos (ou prologues) isolés, dont on ne peut savoir si la pièce correspondante a été perdue ou si elle n'a jamais été écrite. Mais les introitos ont toujours, dans ce théâtre, une certaine autonomie par rapport à la Farsa qu'ils accompagnent et sont, en fait, une petite pièce préliminaire à eux seuls. On peut observer cela dans les prologues de la Farce du forgeron et de la Farce de sainte Suzanne (où sont mis à l'honneur les jardiniers), prologues qui ne se réduisent pas au monologue du Berger présentateur mais sont dialogués, ou encore dans le Prologue des pêcheurs, qui ne se limite pas à des paroles, mais est accompagné d'une action (le personnage distribue à la fin de la pièce du poisson aux personnalités présentes dans le public).

On observera d'abord quels sont les métiers nommés, et à quel moment ils le sont pour la première fois dans la pièce, en quels termes cela est fait, à savoir sous une perspective uniquement louangeuse ou également satirique (nous sommes dans un théâtre comique, ne l'oublions pas). Nous verrons aussi quel est le personnage chargé de dépeindre ce métier, tantôt le Berger présentateur, tantôt, directement, un représentant sur scène de ce corps de métier. Nous nous attarderons spécifiquement sur la technique employée pour mettre en théâtre la profession représentée (description ou représentation sur scène de certains gestes professionnels) et, en particulier, sur la valeur d'expressivité théâtrale de ces figures professionnelles empruntées à la vie sociale.

Pourquoi Sánchez de Badajoz a-t-il choisi de représenter ces six métiers-là ? Il faut préciser d'abord qu'il existe quelques autres mentions

\footnotetext{
- Farsa del molinero (' Farce du meunier') en que entran quatro figuras i un Molinero que sirve de Pastor, muy enharinado, y un Frayle, y un Ciego y su Muchacho que lo adiestra. Habla del Sanctissimo Sacramento del Corpus Christi, p. 403-411.

- Farsa del colmenero (' Farce de l'apiculteur'). Son ynterlocutores un Pastor que se hizo colmenero $y$ un Frayre $y$ un Labrador ; $y$ a de levar el Pastor puesta una máscara de castrar colmenas y una castradera en la mano y una payla con panales en la otra i y a de llevar la payla a la parte de fuera ciertas manchas de lechada, p. 227-241.

- Farsa de Santa Susaña (' Farce de sainte Suzanne ') en que entran seys figuras. A de yr la carreta hecha un vergel, y a la una parte ascondidos dos Viejos con sus varas como juezes, y a la otra parte una muger muy adereçada que es Susaña i y a de estar a la una parte del vergel un mancebo clérigo que es el profeta Daniel y un ángel encubiertos, y un ortolano y un Pastor, p. 423-442.

3 La Farsa de Santa Susaña, ou apparaît l'Ortolano (' le Jardinier '), a été jouée sur une charrette tenant lieu de scène de théâtre mobile, déplaçable d'un coin à l'autre de la ville et servant d'estrade surélevée. Le décor n'est pas totalement absent, mais est réduit à quelques éléments d'atrezzo de base : des brassées de végétaux pour que les vieillards de la Farsa de Santa Susaña puissent s'y cacher, les instruments de travail de l'Apiculteur, etc. Il n'y a pas de véritable décor, mais la mise en scène tire parti de certaines caractéristiques du lieu de représentation. Dans la Farsa del herrero (' du forgeron '), la pièce a probablement été jouée devant un décor réel de forge. Le public de ce théâtre a une composition sociologique composite : dans les quelques représentations privées, il réunit la noblesse locale, les clercs et la hiérarchie religieuse locale; dans les représentations publiques, c'est un public mélangé et varié, où se côtoient la communauté des fidèles et la hiérarchie religieuse. (ex. : l'archevêque Silíceo).
} 
de métiers dans ce théâtre, mais que l'on a choisi ici de parler des pièces et introitos où l'accent est fortement mis sur un métier précis, et en particulier, où il est présent dans le titre (Yntroito de pescadores, Yntroito de herradores, Farsa del molinero, Farsa del colmenero, etc.). Mais dans d'autres Farsas, non étudiées ici, figurent des allusions plus ponctuelles à d'autres métiers : par exemple, dans la Farsa de San Pedro, on trouve une allusion à la profession de pêcheur ${ }^{4}$, mais qui reste très annexe. Une autre fois, dans la Farsa del colmenero, une deuxième profession non annoncée dans le titre, celle de Laboureur, est longuement commentée, et à cet égard, on peut considérer que les six pièces ou prologues analysés mettent en réalité à l'honneur sept professions. Quant à la profession de jardinier, bien que le mot Ortolano (Ortolano, ' maraîcher ', ' jardinier ') ne figure pas dans le titre, elle doit absolument être incluse dans ce recensement en raison du relief qui lui est donné dans la représentation dramatique correspondante. Le critère primordial de sélection de notre corpus a donc été de retenir les professions qui sont mises en théâtre longuement dans la pièce ou le prologue, et dont l'évocation est faite au moins partiellement dans un esprit d'éloge. Il existe en effet aussi une Farsa de la ventera, qui fait partie du petit groupe des farces profanes de cet auteur, pièce qui est entièrement critique à l'égard de cette profession, et qui n'entre pas dans notre corpus, bien qu'elle nous serve d'exemple à opposer aux pièces sélectionnées.

On constate que toutes les pièces répondant à ces critères étaient prévues pour la Fête-Dieu et avaient donc des commanditaires que le dramaturge se devait de flatter. Aucune des pièces de cet auteur consacrées à une profession n'étant jouée pour une autre occasion que cette fête, elles font donc toutes partie du vaste sous-ensemble des farces religieuses, alors que la Farsa de la Ventera, par exemple, appartient à la catégorie des farces profanes. Si l'on examine la forme ${ }^{5}$ prise par ces Farsas de métiers, on constate que l'on a affaire ici essentiellement à des farces dialogales : la seule exception est la Farsa de Santa Susaña, qui est du type " figuratif " et met en scène une scène biblique bien connue, celle de Suzanne et les vieillards. D'ailleurs, à cette exception sur le plan de la forme correspond justement l'absence de mention du métier d'Ortolano dans le titre.

Pour revenir sur le choix des corps de métiers représentés, on serait tenté de penser qu'il reflète simplement le tissu social de la bourgade rurale où vivait ce dramaturge. Pourtant, ce n'est pas seulement en tant que calque de la situation économique environnante que ces corps de métiers figurent dans ces pièces. Pour chacun d'eux, le dramaturge se livre à une exégèse des significations que représentent ces professions sur le plan de la symbolique religieuse, et c'est sur les professions qui permettent une riche lecture typologique, symbolique ou allégorique que se porte son choix, ouvrant de ce fait la voie aux Autos sacramentales qui représenteront l'achèvement de ce mode d'expression.

La mise en valeur de chacune de ces professions commence souvent dès le titre. Cette mention peut être limitée à sa plus simple expression, comme dans la Farsa del molinero, ${ }^{6}$ ou bien comporter une précision

4 Farsa de San Pedro, v. 4-90.

5 Il existe trois types de farces, dans ce thêatre : les farces dialogales, les farces allégoriques et les farces figuratives, qui font une lecture typologique d'un épisode biblique.

6 Ces commentaires concernent les titres qui figurent en tête des Farsas, car, dans la table des matières (tabla), ils sont formulés un peu différemment et de façon plus complète, et souvent ces compléments sont très intéressants dans la perspective qui est la nôtre : Farsa de un colmenero $y$ un labrador que porfían sobre quál de sus o / fficios es mejor, y un frayle que les declara algunas cosas que cada uno / dellos puede notar en su officio, aplicadas al Santisimo Sacramento del / altar.

Farsa de un molinero, en la qual se trata del Santíssimo Sacramento del / Corpus Cristi.

Farsa en que se trata quel officio del herrero es el más útil y necesario / de todos para la vida humana. 
géographique, comme l'" Yntroito de pescadores de tierra de Badajoz ", libellé qui, dans la table des matières, se transforme en : " Prologue des pêcheurs de la terre de Badajoz où l'on loue le métier de pêcheur et I'on décrit les étangs et les poissons du Guadiana dans la région de Badajoz ", modification qui reflète bien la nature de ce texte dont on verra qu'il est pétri de référence topographiques locales. Mais le titre, comme il est de mise dans le théâtre du XVIe siècle, contient souvent, aussi, des indications concernant le jeu des acteurs ou l'apparence du personnage : au début de la Farsa del molinero, on lit que figure dans la pièce un meunier " tout enfariné "7 ; ou encore, dans la Farsa del colmenero, que le personnage de l'Apiculteur " doit porter un masque pour la récolte du miel, un couteau à désoperculer dans une main et, dans l'autre, une bassine avec des rayons de miel ${ }^{18}$. La précision est parfois poussée très loin : " la bassine doit avoir des taches de lait de chaux à l'extérieur "9. Les indications ne se limitent pas au maquillage et au costume des personnages, mais dictent parfois toute une gestuelle : " Farce du maréchal-ferrant, où figurent trois personnages : un Maréchal-ferrant maniant le marteau près de sa forge et un Berger et un Pèlerin "10. La mise à l'honneur du métier conduit à placer le nom qui le désigne en tête de liste de personnages, même si ce n'est pas le représentant de ce métier qui parle en premier. Il est vrai qu'à défaut de prendre la parole en premier, le Forgeron, par son jeu de scène, est l'élément scénique sur lequel s'ouvre la pièce, puisque la première prise de parole, celle du Berger, est une réaction au spectacle du Forgeron frappant sur son enclume. Seul le Jardinier, dans le prologue de la Farsa de Santa Susaña, est cité en fin de bloc didascalique, car, bien qu'il prononce une part importante du dialogue, de toute évidence, il ne peut voler la vedette aux personnages principaux que sont suzanne et les Vieillards. Mais, comme pour compenser cette relégation du Jardinier à la fin de la didascalie, le début de celle-ci est consacré au décor représentant le jardin :

Farce de Sainte Suzanne dans laquelle se trouvent six figures. La charrette doit avoir l'aspect d'un verger, et d'un côté, cachés, deux vieillards avec leur bâton comme en ont les Juges, et de l'autre, une femme très richement vêtue qui est Suzanne; et d'un côté du verger doit se trouver un jeune prêtre qui est le Prophète Daniel et un Ange, dissimulés, et un jardinier et un Berger, lequel commence à dire ${ }^{11} \ldots$

Ces didascalies initiales, malgré leur brièveté, donnent déjà un avant-goût des éléments concernant la nomination des métiers et de la façon dont ils seront mis en théâtre dans le prologue et dans le corps de la farce.

Après les titres, voyons maintenant de quelle façon sont évoquées ces professions dans les textes eux-mêmes.

Dans l'Yntroito de herradores (' Prologue des maréchaux-ferrants '), on trouve une accumulation de désignations : outre le mot herrador, il y a une périphrase, el oficio del herrar (' le métier de ferrer '), une mention de Dieu qualifié de herrador divino (' le divin maréchal-ferrant '), et un pluriel herradores, qui rappelle que le commanditaire de la pièce est une

Yntroyto para los pescadores, en que se alaba el oficio del pescar y / se cuentan los charcos y pescados que tiene Guadiana en término de Ba / dajoz.

Yntroyto para herradores, en que se alaba el officio del herrar, p. 51-53, op. cit.

7 " Un molinero muy enharinado ".

8 " ha de levar puesta una máscara de castrar colmenas y una castradera en la mano y una payla con panales en la otra ".

9 " y a de llevar la payla a la parte de fuera ciertas manchas de lechada ".

10 "Farsa del herrero en que entran tres figuras : un Herrero martillando junto a su fragua, y un Pastor $y$ un Romero ".

11 "Farsa de Santa Susaña en que entran seys figuras. A de yr la carreta hecha un vergel, y a la una parte ascondidos dos Viejos con sus varas como juezes, y a la otra parte una muger muy adereçada que es Susaña; $y$ a de estar a la una parte del vergel un mancebo clérigo que es el profeta Daniel y un Ángel encubiertos, y un Ortolano y un Pastor, el qual comiença a dezir... " 
entité collective. À cela, il ne s'ajoute pas moins de deux occurrences du mot herrería (forge), onze mentions du verbe herrar et cinq de frenar (' mettre un mors '), puisque le maréchal-ferrant est évoqué à travers ses deux productions principales, le fer-à-cheval et le mors du cheval. On voit que le dramaturge ne lésine pas sur l'effet de répétition pour donner plus de présence à la profession qu'il souhaite mettre en valeur. L'effort d'expressivité, notons-le, se manifeste surtout par la présence de verbes décrivant la gestuelle ou la production de la profession.

L'Yntroito de herradores ne met pas en scène la gestuelle du maréchal-ferrant, mais, en guise de substitut, une dérivation de la gestuelle de l'animal ferré, traduite sous la forme de gesticulation du Berger présentateur qui s'exclame : " Oh, si je pouvais avoir des souliers de fer pour danser cent ans jusqu'à en tomber par terre ! [...] "12.

Dans la Farsa del herrero, le geste du Forgeron est mis en scène dès le début du texte et sert à compléter le décor verbal que dresse le Berger présentateur en décrivant le spectacle de la forge. La mise en scène spectaculaire de la terreur du Berger face à ce personnage sombre et menaçant, qui se détache sur fond de feu et qu'il prend pour un diable, décuple le pouvoir évocateur du geste du Forgeron.

Dans la Farsa de pescadores, la triple mention de la profession dans les premiers vers cède le pas à un autre type de marée verbale, celle de l'énumération des lieux de pêche qui inscrit profondément la profession dans un paysage bien connu des spectateurs, celui des environs de Talavera. Cet enracinement géographique s'accompagne de l'évaluation de la qualité des coins de pêche évoqués, et permet au locuteur de faire étalage de toute sa compétence. Ainsi, à travers la technique du décor verbal, bien connue dans le théâtre ancien espagnol qui était dépourvu de décor matériel, le dramaturge réussit, de surcrô̂t, à suggérer la haute valeur professionnelle des pêcheurs. L'énumération de pas moins de dix-sept espèces de poissons de rivière, par la science qu'elle suppose, concourt au même effet, tout en offrant l'image providentialiste d'une nature abondante qu'un Dieu généreux offre aux hommes. Cette insistance sur les poissons ne semble pas être liée à leur valeur de symbole à l'époque du premier christianisme. Il ne s'agit pas seulement d'une évocation encyclopédique ou allégorique, lorsque le personnage s'exclame : " Moi, je connais à fond les poissons petits et gros "13, mais du reflet authentique de la connaissance passionnée d'un milieu naturel proche. La profession du pêcheur est évoquée quadruplement, à travers la dénomination du métier, à travers une gestuelle, la pêche au lancer (" en Don Miguel he lançado ", v. 97), à travers le matériel utilisé : "filets, nasses, casiers, bourriche "14, et aussi à travers un décor multiple mais dessiné avec une précision de carte d'état-major où les paysages les plus variés apparaissent : vallée, rochers, gorge, ravin, mare, étang, gué, lagune, embouchure, bassin, marais, déversoir ${ }^{15}$. Même la végétation locale est précisée dans ce tableau complet de la profession de pêcheur : aunes, frênes, lierre ${ }^{16}$ entourent les lieux de pêche. Le dramaturge en dressant cette carte des coins de pêche de sa région réussit, par sa précision, à impliquer les spectateurs qui doivent forcément connâ̂tre l'un ou l'autre de ces parages, et prend soin de dessiner un paysage affectif avec des termes subjectifs (la gorge du Frêne des Corbeaux est qualifiée de " belle "17), ou encore avec des exclamations louangeuses comme " [...] qui peut oublier pareille chose ? "18 et des toponymes pleins de

\footnotetext{
12 " i Oh, quién pudiera tener / unos çapatos de hierro / para baylar [...] / cien años hasta caer ! ", v. 1-4.

13 " Yo tengo bien conocidos / pescados y pescadillos ", v. 105-106.

14 " redecillas, / xudras, nasas, esqueros ", v. 117-118.

15 " val ", " peñas ", " colada ", " hoyo ", " charco ", " charca ", " vado" ; " albufera ", "boca ", " bacinete ", " laguna ", " henchidero ".

16 "Alisos", "fresnos ", "yedra".

17 " y la Colada y su yra / del Fresno de Cuervos, bella ", v. 45-46 ; " charca Hermosa es tamaña ", v. 87 .

18 " [...] ¿ quién lo tira ? ", v. 48.
} 
couleurs (" Charca Branca de Pesquero ", v. 81), le tout aboutissant à un portrait en mouvement de la profession de pêcheur, particulièrement adapté à l'esthétique du théâtre qui laisse toujours une grande part à la gestuelle, représentée ou évoquée.

Dans la Farsa del colmenero, le nom de la profession d'apiculteur n'est pas nommé de façon aussi insistante, mais, en revanche, son activité est décrite par le menu à travers le récit familier d'une anecdote qui tourne mal pour lui : pour répondre à une commande des jeunes filles de son village qui lui demandaient de la cire d'abeille récente pour leur maquillage, il va récolter quelques rayons de ses ruches, mais est victime d'une attaque de ses abeilles. La profession, ici, il faut le reconnâtre, n'est pas vraiment décrite dans une situation de compétence, mais plutôt d'accident survenu de façon exceptionnelle chez un professionnel pourtant fort bien protégé et dont l'équipement technique est complaisamment présenté sur scène et pas seulement décrit.

C'est un peu le même phénomène dans la Farsa del molinero, où l'on n'insiste pas beaucoup sur le nom de la profession (encore que, au moment où le dramaturge la désigne, il réussisse à renforcer astucieusement la mention de cette profession en faisant dire au Meunier qu'il est " moulu de fatigue ${ }^{19}$ ), mais où l'activité du meunier est longuement évoquée sur scène de façon satirique. Ce mode de mise en théâtre qui s'affirme dès la didascalie initiale où le Meunier apparâ̂t enfariné de façon comique se poursuit par la description, après d'autres gestes professionnels, du mouvement de bras que fait le Meunier pour dérober un peu de blé de chaque sac qu'on lui apporte. Le dramaturge se fait l'écho de la réputation de voleur que l'on attribuait traditionnellement aux meuniers et montre, à côté de la compétence professionnelle du personnage, son habileté dans l'art du larcin. La description des geste professionnels est précise : " Dès que je ramène et prends et relève et remets à moudre le grain, je ne peux plus tenir debout et n'ai plus que la peau sur les os"20. C'est par des récits fragmentaires de petites scènes populaires que se complète la vision du métier de meunier. Le personnage relate, par exemple, ses conversations grivoises avec les commères qui viennent faire moudre leur grain ${ }^{21}$ ou encore les journées d'été où les gens viennent pique-niquer au moulin. Ces scènes de la vie quotidienne donnent lieu à l'emploi de procédés dialogiques dans l'introito, où les échanges verbaux entre le Berger et les commères apparaissent cités en style direct, comme une petite scène de théâtre insérée dans le prologue : la peinture de la vie sociale, loin d'affaiblir la thêâtralité, la renforce.

L'originalité de la désignation du métier de jardinier tient à ce que, dans la Farsa de Santa Susaña, ce personnage est d'abord désigné, dans la bouche du Berger présentateur qui commente au public l'arrivée de ce nouveau personnage, sous le nom de cavador (" cavador es el rapaz ", v. 128 ; cavador : ' celui qui creuse la terre ', le ' terrassier ') ; autrement dit, la profession est désignée de façon approximative, dans le plus pur style d'expression du Berger nä̈ (Pastor Bobo). Mais si le mot cavador ne décrit pas exactement la profession du Jardinier, du moins décrit-il l'un de ses gestes les plus fréquents : piocher la terre. La

19 " ¿ Cree que ser molinero / es comportable tormento ? / i Dios, qué molido me siento ! ", v. 33-35.

20 " Desque ayunto y cojo y pico / y torno a echar a moler, ya no me puedo tener / ni queda de mí çatico ", v. 41-44.

21 " Llas mugeres con maldades / como campanas, i dan, dan !, / diz que le sal poco pan ; / i escuchá qué bovedades ! / Yo acudo con las verdades; /digo, desque las escucho : / Andá, andá, que cernís mucho / para her bollos abades ". / Algunas se van riendo, / otras me dizen vellaco; / palabras no rompen saco, / hago que no las entiendo. ", v. 65-76 : ' Les femmes avec leurs méchancetés, / comme des cloches vous sonnent aux oreilles à toute volée ! /Écoutez-moi ces âneries ! / Moi je leur dis leurs quatre vérités ; / je dis, quand je les entends : / " Allons, allons, vous tamisez beaucoup / pour faire des brioches pour le curé. [le geste de tamiser avait des connotations érotiques] / Les unes s'en vont en riant / les autres me traitent de voyou ; /les mots n'ont jamais tué personne, / je fais comme si je ne les entendais pas ". 
désignation de ce métier commence donc par un geste au fort pouvoir graphique, chargé de théâtralité. Mais cette stratégie d'évocation est bientôt remplacée par le processus d'énumération des fruits du travail, énumération qui dépasse largement la liste encyclopédique des poissons qui figurait dans l'Yntroito de pescadores. Là encore, cette litanie permet de faire défiler tous les noms de fruits et de légumes connus et sert non seulement à exprimer l'excellence du savoir technique des jardiniers, mais déploie toutes les richesses mises au service de l'homme par un Dieu bienveillant. La liste est donnée par catégories (fruits, légumes ${ }^{22}$ ) et par saisons, ce qui confère à ce fragment de la pièce une impression d'ordre et de monde mâ̂trisé, destinée à traduire tout autant l'harmonie de l'univers que l'excellence professionnelle des jardiniers.

\section{Un éloge simple mais théâtralisé}

Alors que, dans l'Yntroito de pescadores, l'éloge se faisait de façon implicite en déployant la connaissance parfaite qu'avait le personnage des lieux de pêche, la louange prend généralement, dans ces pièces, une forme plus explicite et plus conventionnelle. La plupart de ces textes se livrent à un éloge simple et sans nuage du métier évoqué, comme celui que l'on trouve dans l'Yntroito de herradores : " Il est de haute utilité, à mon avis, le métier de forgeron, qui permet aux bêtes de durer autant qu'elles le peuvent ${ }^{23}$. Ce type d'éloge élémentaire et conventionnel est toutefois soigneusement théâtralisé à travers la gesticulation décrite par le Berger, au moyen de termes hyperboliques visant à rappeler la solidité d'un fer-àcheval (v. 1-4) : ces souliers ferrés sur lesquels fantasme bruyamment le Berger en entrant en scène lui permettraient, dit-il, de danser pendant cent ans, jusqu'à en tomber par terre.

\section{Un éloge à deux voix}

Lorsque le personnage du Berger présentateur est utilisé sous une version plus sérieuse de son personnage, comme dans la Farsa de Santa Susaña, cet éloge peut se faire à deux voix, le Berger dialoguant alors avec le Jardinier dans un long duo ${ }^{24}$ de plus de cent vers, (v. 129-240) où les répliques de l'un s'enchaînent aux répliques de l'autre, dans la plus grande harmonie, tant au niveau du contenu des idées exposées que de la structure syntaxique des répliques. L'impression reçue est celle d'un seul et même discours, rendu plus amène toutefois par une présentation à deux voix. Mais, si les deux personnages sont en symbiose pour chanter les mérites de la profession, ils s'opposent soudain, peu après, sur la manière de faire l'éloge du travail. Le Jardinier, en effet, non seulement fait l'éloge de sa profession, mais se réjouit du travail qu'elle réclame et finit par se faire le chantre des vertus du travail en général ${ }^{25}$. À l'opposé, le Berger, que la tradition littéraire dote d'une paresse sans égale et d'une propension constante à dormir, s'insurge contre cette théorie et vante l'oisiveté. Mais cette opposition comique n'est qu'un procédé dialogal destiné à donner au Jardinier l'opportunité de mieux

22 " [...] guindas, cerezas, mançanas / ciruelas de mill tenores : / çeremeñas y albocores, / moras y peras galanas / y las albérchigas sanas, / priscos y malacotones / y duraznos a montones / y membrillos y granadas ", etc. : ' guignes, cerises, pommes, prunes de mille espèces, poires muscadelles, abricots, mûres et belles poires, et saines alberges, priscos [autre espèce d'alberge] ou pêches ', v. 145-220.

23 " Gran provecho es a mi ver /: ell oficio del herrar / que a las bestias haz durar / quanto basta su poder ", v. 5-8.

24 " Duo " dans le sens où l'entendent A. Ubersfeld et M. Vinaver : dialogue fusionnel où les déclarations des deux personnages vont dans le même sens. Ce type de dialogue permet des répliques grammaticalement incomplètes, où la phrase de l'un s'appuie sur celle de l'autre. Ce passage de la Farce de Sainte Suzanne est si fusionnel que, dans l'édition de Frida Weber de Kurlat, il se produit une hésitation dans l'attribution d'une réplique à ces personnages. 25 Cette thématique, liée à la scène biblique représentée, est induite par l'idée que c'est l'oisiveté qui est à l'origine du péché des deux vieillards. 
étayer l'argumentation de son éloge du travail (v. 229-252). Ceci montre que les modalités de construction du dialogue (ici, successivement, le duo et l'opposition) figurent aussi parmi les éléments sur lesquels joue le dramaturge pour exposer les caractéristiques d'une profession devant le public. L'exemple cité montre aussi que l'éloge des professions est accompagné de réflexions moralisantes tout autant que de l'exploitation des symboles religieux évoquée plus haut.

\section{Caricature et éloge}

Pour en revenir à l'éloge du métier, on est toujours un peu étonné de constater que ce dramaturge n'hésite pas à faire voisiner la louange avec des éléments burlesques et même satiriques.

L'Apiculteur, on l'a vu, est représenté dans un moment peu glorieux de son métier, où les abeilles, plus habiles que lui, réussissent à le piquer. Le Meunier, lui, se dépeint comme objet des plaisanteries des femmes qui viennent au moulin. Quant au Forgeron, après avoir parlé fort doctement au Berger ignorant, il est néanmoins exposé aux réflexions insolentes de ce même Berger :

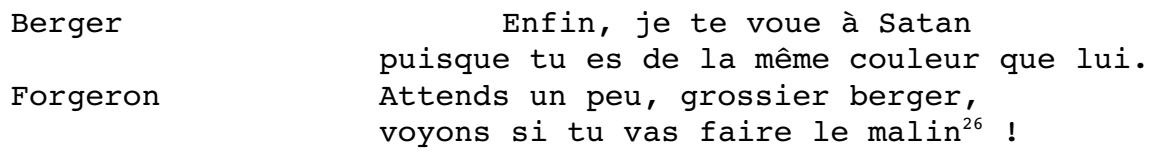

Ainsi, le dramaturge mêle sans préjugé l'éloge et la satire des professions.

Dans la Farsa del herrero, la description burlesque du Forgeron, comparé à un diable couvert de suie s'agitant devant les flammes de l'enfer, permet une caricature de la silhouette de l'artisan, mais en limitant prudemment cette caricature à l'aspect physique : il ne faudrait pas prendre le risque de ridiculiser vraiment le Forgeron et sa profession devant le public. La caricature consiste, par exemple, à affubler ce personnage du vocable de escuerzo (' crapaud ', ou ' grand échalas '). Cette peinture de la gesticulation du forgeron sert à mentionner à diverses reprises les outils de travail : le feu, le marteau ${ }^{27}$, les tenailles ${ }^{28}$. Dans le dialogue qui s'instaure entre le Berger et le Forgeron, ce sont ensuite les objets (ou parties d'objets) produits qui sont mentionnés, comme la dent d'un outil (gajo) ou l'arbalète (ballesta) : " Berger : Vous savez faire une dent d'outil. / Forgeron : Et même aussi une arbalète ${ }^{29}$ ".

La caractérisation professionalisante n'est donc pas perdue de vue par le dramaturge, même au plus fort de la caricature. Cependant, le soin de faire une louange en bonne et due forme de la profession est laissé au Forgeron lui-même : il était difficile de placer un éloge crédible dans la bouche du Berger, ridiculisé sur scène par sa couardise, sa bêtise et ses craintes superstitieuses. L'éloge de la profession a d'autant plus de poids que le Forgeron, dans cette pièce, se trouve revêtu du rôle de personnage du savoir, qui, face à l'obscurantisme du Berger, donne des explications claires et solides :

Forgeron Tu n'as jamais vu de forgeron?

Approche, tu verras, n'aies pas peur.

Je suis un homme comme toi ${ }^{30}$.

26 Pastor : "En fin, doyte a Satanás, / pues que tienes su color ". / Herrero : "i Esperá, çafio pastor ! / i Veamos si burlarás ! ", v. 145-148.

27 V. $11,37,47$.

28 V. 52 .

29 Pastor : " Vos sabeys hazer un gajo ". / Herrero : " Y aun también una ballesta ", v. 5960 .

30 Herrero : " ¿ Tú nunca viste herrero? / Llega y verás, no te alteres, / que hombre soy como tú eres ", v. 45-47. 
Mais si, dans cette pièce, le dramaturge a forcé la note sur la lâcheté du Berger, c'est pour mieux faire ressortir le calme, la force et la tranquille assurance du Forgeron. Ce rôle de faire-valoir du Berger se retrouve dans la mise en dialogue de l'éloge du Forgeron sous la forme d'une série de questions/réponses, dans le plus pur style catéchétique :

$\begin{array}{ll}\text { Berger } & \text { À quoi servent les forges ? } \\ \text { Forgeron } & \text { Je vais te donner la réponse. } \\ & \text { Cet art, mon frère, ingénieux s'il en est, } \\ & \text { au sein des activités humaines, } \\ & \text { se situe parmi les plus universels } \\ & \text { et il est, entre tous, le plus productif }{ }^{31} .\end{array}$

L'éloge du métier de forgeron est construit, comme on l'a vu dans d'autres exemples déjà, sur un schéma longuement énumératif, non pas seulement pour citer de façon plus complète encore les objets produits, mais en se plaçant sur un registre qui concerne tout le tissu social, pour brosser un tableau de toutes les professions qui, dans leur exercice, ont recours à l'emploi d'instruments fabriqués par le forgeron :

\begin{tabular}{|c|c|}
\hline Forgeron & $\begin{array}{l}\text { N'importe quelle réalisation utile } \\
\text { à notre subsistance } \\
\text { prend ses outils de cet art } \\
\text { sans lequel rien n'existerait }{ }^{32} \text {. }\end{array}$ \\
\hline
\end{tabular}

Ce passage est d'ailleurs, dans toute l'œuvre de cet auteur, celui où l'on trouve la plus longue énumération de professions. Ainsi, l'éloge du Forgeron se transforme en un condensé de celui qu'on pourrait faire des autres secteurs d'activité : le premier terme employé est celui d'obrero (v. 77), terme générique, suivi de termes plus spécifiques, albañires, canteros (v. 82), carpinteros (v. 83), arte militar (v. 89), çapateros et lanificios (v. 94), la agricultura (v. 105). Chacune de ces activités est mise en scène dans ses gestes et son outillage, et renvoie, par effet de miroir, à la production du forgeron :

Forgeron [...] parce que pour le premier travail

sur la laine, qui consiste à la récolter, et pour prélever le cuir,

on se sert de couteaux et de ciseaux,

ensuite, viennent le peigne et la carde,

et même le rateau en fer

qui sert à mettre en tas le lin $[\ldots]^{33}$.

La culture du blé et de la vigne permettent de parler des outils : azada, podón, azadón, reja, rejada, aguijón, sacho, hoçe, guadaña. Ces tableaux imbriqués au sein même de l'éloge du Forgeron, en soulignant la prééminence de sa profession sur les autres, représentent en eux-mêmes un élément valorisant. Cet aspect va de pair avec la prééminence donnée dans le dialogue au rôle du Forgeron, à qui il échoit de réciter le plus long texte de la pièce.

En comparant avec la Farsa de la ventera (' Farce de l'aubergiste '), à laquelle nous avons fait allusion au tout début de ce travail, et qui est uniquement satirique, on peut formuler l'hypothèse que la présence d'un contenu purement élogieux, ou au contraire mixte, mêlant éloge et satire, est induite par la respectabilité de la profession évoquée. Certaines

31 Pastor : " ¿ Para qué van herrerías ? "/ Herrero : " Quiérote dar la respuesta. / Esta arte, hermano, yngeniosa, / en las artes humanales / es de las más generales / y en todas más abundosa ", etc., v. 64-123.

32 Herrero : " Qualquier obra provechosa / a nuestros mantenimiento / deste arte toma estrumentos, / sin la qual no se haz cosa, v. 69-72.

33 Herrero : "Porque a la cosa primera / de la lana ques coxella, / y al cuero que se devella / sirven cuchillo y tigera, / después peyne y cardadera, / y aún el lino puesto en cerro / con un rastrillo de hierro [...] ", v. 97-104. 
professions, comme celles de meunier ou d'aubergiste, traînent avec elle une réputation sulfureuse. Mais si l'Aubergiste reçoit un traitement littéraire uniquement satirique, en revanche, le Meunier, convoqué sur scène par Diego Sánchez en raison du lien symbolique existant entre la farine, le pain et l'hostie, se voit appliquer une formule mixte, où éloge et satire font bon ménage. Ce que le spectateur moderne pourrait interpréter comme un manque de cohérence du personnage n'est en fait qu'une conséquence de l'esprit pragmatique dans lequel est écrit ce théâtre : l'éloge permet d'insérer la pièce dans son contexte social et financier, et la satire conjointe reprend les formules à succès de la tradition populaire, sans que cette apparente contradiction pose un problème à 1 'époque.

Face à la tranquille satisfaction du Jardinier qui affirme : " C'est du travail que d'être jardinier, mais cela nourrit son homme "34, la déclaration d'une identité professionnelle s'exprime souvent, dans ces farsas, sous forme de plainte.

L'Apiculteur de la Farsa del colmenero se plaint bruyamment des difficultés de son métier:

Berger Je jure sur le Dieu véritable

qu'il me faut suer pour manger ;

[...]

Je suis devenu apiculteur

pour vivre tranquille,

mais, là aussi, il y a des piqûres,

c'en est un vrai cauchemar ${ }^{35}$.

Le meunier de la Farsa del molinero est tout aussi éloquent pour se plaindre de sa condition :

Meunier

Vous croyez qu'être meunier

est une souffrance supportable?

Mon Dieu! Je suis tout moulu,

plus qu'un âne de bât ;

dans aucune fête ni réjouissance,

jamais je ne peux m'arrêter ${ }^{36}$.

De la plainte sur la vie et sur la profession à la protestation sociale, il n'y a qu'un pas. La déclaration d'appartenance professionnelle se conjugue-t-elle avec l'affirmation d'un sentiment d'insatisfaction sociale ? Le théâtre de Sánchez de Badajoz n'est certes pas un théâtre contestataire de l'ordre social, bien au contraire, et cependant la déclaration de profession faite par certains personnages donne lieu à des embryons de protestation. La plainte de l'Apiculteur sur la difficulté de son métier est précédée d'une dénonciation de l'injustice sociale :

Berger Il faut bien que nous puissions tous manger, mais comme il n'y a pas beaucoup de sacs [de blé], tout est consommé par les voyous qui mangent sans avoir à récolter ${ }^{37}$.

Pour juger à leur juste valeur ces protestations, il ne faut pas oublier que, sous les divers métiers représentés, se manifeste de façon résurgente la nature du personnage littéraire polyfacétique qui leur sert

34 " Trabajo es ser Ortolano, / mas mantiene a quien lo usare ", v. 137-138.

35 Pastor : "Yo juro a Dios verdadero /que no como sin sudar ; / [...] / Yo me hiçe colmenero / por bivir a descansadas, / mas también alli hay picadas / que es un gran rreñegadero ", v. 916 .

36 Molinero : " ¿ Cree que ser molinero / es comportable tormento ? / i Dios, qué molido me siento !, / más que un asno de recuero; / en fiesta ni festigero / nunca ell ombre a de parar ", v. 33-38.

37 Pastor : "Todos emos de comer, / mas, aviendo pocos sacos [de trigo] /todo llo gastan vellacos / que comen sin 110 coger. ", v. 5-8. 
de support. Le dramaturge met en scène des métiers, mais c'est le Berger, non dans sa fonction sociale, mais dans sa fonction littéraire traditionnelle de personnage gesticulateur, extraverti, insolent, querelleur, obtus, paresseux et gourmand, qui affleure sous les divers masques qu'il adopte. La protestation sociale endossée par ce personnage, au même titre que son éloge de la paresse, doivent donc être relativisés en considérant les caractéristiques littéraires dont il est pétri. Le thème du masque est tout de même l'occasion de poser ici la question de la désignation des métiers dans les didascalies donnant le nom des personnages. Le masque arboré dans la Farsa del colmenero par le personnage de l'Apiculteur, en guise d'accessoire identificateur, est aussi un masque de thêâtre : comme dans le " vieux proverbe de la SNCF " qui dit qu'" Un train peut en cacher un autre ", dans ce théâtre un personnage en cache un autre. Dans une dramaturgie qui, il faut le signaler, n'était pas toujours rigoureuse dans ce genre de notations didascaliques, on observe un flottement tout de même assez révélateur, dans les noms de personnages inscrits en marge des répliques. Dans la Farsa del colmenero, c'est bien l'Apiculteur lui-même qui décrit sa profession, mais cependant, dans la marge, en guise de nom de personnage, figure la dénomination de Pastor, Berger. Le dramaturge semble donc avoir fait primer la fonction sur l'identité : le Berger étant le personnage dont la fonction habituelle est de prononcer le prologue, on lui laisse son identité de Berger de théâtre, quel que soit le métier représenté.

Une autre didascalie exprime parfaitement combien le mot Berger est connoté par l'univers du théâtre : elle précise que, dans la liste de personnages, figure " un meunier qui sert de Berger " (" un Molinero que sirve de Pastor "). C'est donc, dans cette pièce, un personnage de Meunier qui récite l'introito, chose que fait habituellement le Berger. Cependant, et peut-être justement en raison de la précision donnée dans la didascalie, " un Molinero que sirve de Pastor ", le nom de métier apposé en marge pour désigner le personnage est bien celui de Molinero, selon une logique de présentation totalement à l'opposé de ce qui se passe dans le texte précédemment cité. Est-ce à dire que ce personnage de Meunier n'a plus rien à voir avec le Berger qui lui sert de substrat dramatique ? Ce n'est pas tout à fait le cas ; le dramaturge continue à afficher ce lien, par un procédé qui a l'avantage de ne pas rompre la fiction : le Meunier, dans les propos qu'il tient dans le prologue, ne se contente pas de commenter son état actuel de meunier, mais également sa profession précédente, évoquée avec nostalgie, et qui n'est autre que celle de... berger :

Meunier

On change mille fois de métiers

pour courir après ce terrible argent ;

j'étais auparavant berger,

je vivais bien plus à mon aise ${ }^{38}$.

La mobilité sociale sert ici de prétexte pour donner une apparence de vraisemblance au remplacement du Berger présentateur traditionnel par un autre personnage ${ }^{39}$. De même, l'Apiculteur rappelle qu'il est " devenu " apiculteur : "Yo me hize colmenero ", (v. 13), élément qui figure dans le dialogue, mais était déjà annoncé dans la didascalie initiale, " un Pastor que se hizo colmenero ".

Le comble de la mobilité sociale dans cette désignation des noms de métiers est atteint dans l'Yntroito de pescadores, où le Berger énonciateur dit exercer la profession de Berger, mais exprime le regret de ses deux professions précédentes, de pêcheur et d'apiculteur, et prévoit même de

38 Molinero : "Muda el hombre mil oficios / tras este negro dinero ; / solía ser ovegero, / bevía [= vivía] más a mis vicios ", v. 9-12.

39 Remarquons au passage qu'une autre solution pour faire s'exprimer un personnage de métiers dans le prologue consiste à contruire le prologue non comme un monologue, mais comme un dialogue entre celui-ci et le Berger : c'est la solution adoptée dans la Farsa de Santa Susaña. 
reprendre, dans un futur proche, le métier de pêcheur :

Berger

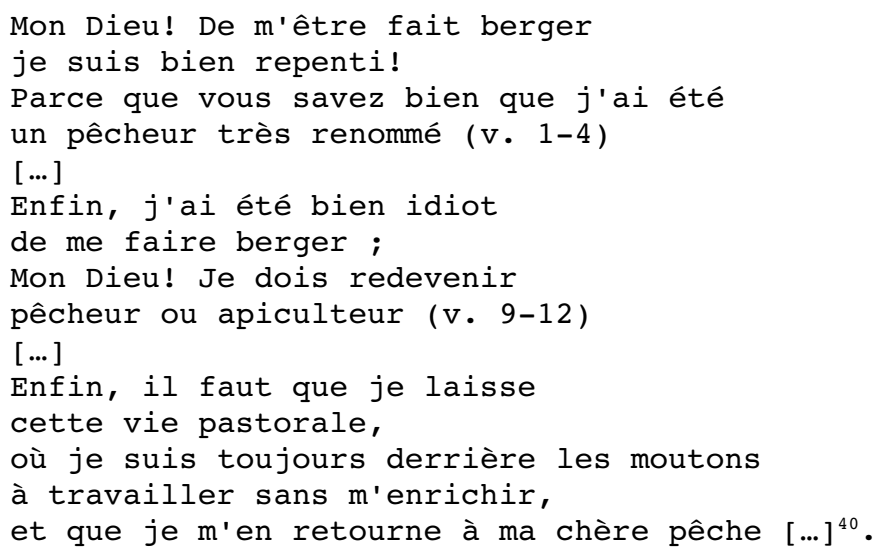

Ces multiples exemples montrent que dans ces pièces consacrées aux métiers, la mention de ceux-ci est fréquemment accompagnée d'allusions à la mobilité professionnelle, sans qu'on puisse juger si c'est pour refléter une réalité sociale ou pour évoquer les transformations du personnage locuteur.

Le Berger de ce théâtre est un faire-valoir des professions mises en scène, qu'il les représente lui-même directement ou qu'il leur serve d'interlocuteur dialogal. Dans tous les cas, il est le médiateur indispensable à la représentation des professions : il n'y a pas de profession représentée dans ce théâtre sans Berger présentateur pour la mettre en scène. Mais ce personnage, véritable moteur dialogal de la pièce, fonction qu'il a héritée des débuts du théâtre castillan où il accueillait les Rois Mages par le " Quem quaeritis ? ", ne reflète-t-il donc jamais sur scène sa propre profession de berger? Déjà, dans les quelques textes que nous avons vus ici, certains aspects concrets du métier de berger apparaissent, en contraste avec les métiers convoqués sur scène. Mais d'autres exemples montrent un stade d'élaboration plus complexe de l'image du métier de berger, comme la Farsa de Ysaac. Dans cette pièce, où est dramatisé l'épisode biblique pendant lequel Jacob, le cadet, détourne à son profit la bénédiction de son père Ysaac, au détriment de son frère aîné, Ésaü, le Berger ponctue la représentation de commentaires exégétiques que l'on peut qualifier de "supradialogaux ", puisqu'ils ne s'insèrent pas dans le dialogue des personnages bibliques, mais s'adressent aux spectateurs, par-dessus le dialogue de la fiction biblique, pour commenter la scène et en tirer divers enseignements moraux et dogmatiques. Ce système de construction dramatique, où le personnage du Berger est complètement extérieur au noyau dramatique biblique, est soudain remplacé, au milieu du texte, par l'insertion d'un bref épisode comique traditionnel, élaboré sur le schéma bien codifié de la dispute (riña). Au cours de cet épisode, le Berger commentateur enfreint de façon très moderne les règles de vraisemblance du temps dramatique et fait irruption au milieu de la scène biblique au moment où Jacob apporte dans ses bras deux chevreaux. Le Berger s'exclame alors que les deux animaux lui appartiennent et que Jacob les lui a volés (v. 131-155). Cette petite scène comique intercalée utilise diverses caractéristiques traditionnelles du Pastor Bobo (impulsif, irréfléchi, etc.), mais surtout, et c'est cet aspect qui nous intéresse aujourd'hui, elle montre le personnage en train de réagir sur scène par rapport à sa profession de berger. L'intérêt de ce passage, outre son

40 Pastor : " i Dios, que de herme pastor / yo me hallo arrepentido ! / porque ya sabéis que he sido / muy nombrado pescador, v. 1-4. [...] / En fin, yo hu majadero /en ovejero me her ; / i Dios, que he de tornar a ser / pescador o colmenero !, v. 9-12. [...] En fin, yo quiero dexarl esta vida pastoriega, / que ando a rrabo de borrega / trabajando sin medrar, / y bolverme a mi pescar. ", v. 33-37. 
originalité, est que le Berger -fait très rare dans ce théâtre- y fait une allusion au tissu social qui l'entoure, concrètement au mâ̂tre qui l'emploie pour garder ses troupeaux : " J'ai un mâ̂tre qui est là pour les manger "41. Un point qui différencie radicalement la désignation de la profession de berger des autres métiers précédemment commentés, c'est que le Berger est un personnage dont on souligne fréquemment la dépendance économique et le bas niveau social, éléments qui sont en harmonie avec ses autres caractéristiques théâtrales de personnage naïf, toujours dans l'erreur face aux divers personnages du savoir, et de personnage constamment rabaissé par ses interlocuteurs savants, qui le traitent de bon à rien (perdimiento). Dans la Farsa moral, le choix du Berger pour représenter la figure du Mal (Nequiçia, vestido como simple pastor, didascalie initiale), est révélateur à cet égard; donc si le métier de berger est mis en scène dans ce théâtre, c'est souvent pour en déplorer la dureté et pour en suggérer la position hiérarchique très humble, perspective qui n'est généralement pas celle des évocations des autres métiers (exception faite, on l'a vu, du Meunier, qui fait allusion aux misères de sa profession épuisante et aux poursuites des officiers de justice dont il est victime).

On peut souligner, pour conclure, que cette mise en valeur des métiers sur la scène est exceptionnelle dans le théâtre de cette époque. On peut prendre comme point de comparaison le Códice de Autos Viejos, recueil anonyme daté de 1575, donc légèrement postérieur, mais dont les quatrevingt-seize pièces ont pu, en grande partie, avoir été écrites à la même époque que celles de Sánchez de Badajoz : on trouve dans ce recueil une seule pièce qui fasse allusion à un métier, celui de semeurs (sembradores), et d'ailleurs dans une perspective allégorique, et sans éloge du métier ${ }^{42}$. Il y a donc bien, dans le théâtre de Sánchez de Badajoz, une originalité certaine dans cette mention systématisante des corps de métiers. Mais, en bon dramaturge, l'auteur ne se contente pas d'en faire un passage obligé destiné à remercier les professions commanditaires de la représentation ; il réussit à en tirer d'intéressants effets de théâtralisation et d'exploitation dogmatique, en particulier pour souligner la providence divine.

La surabondance descriptive qui caractérise ces passages consacrés aux métiers, outre les deux motivations déjà signalées (exploitation symbolique et expression de l'excellence professionnelle), semble correspondre aussi à la puissance d'une entité collective, celle des corporations, qui, à l'égal de ce que fait la noblesse locale, paie les frais de représentations. Tout comme la nomination des métier sur les listes d'enregistrement, ces pièces permettent de renforcer le sentiment d'appartenance à une communauté. Ainsi, à travers ces pièces, la Fête-Dieu ne glorifie pas seulement l'Eucharistie, mais la profession des commanditaires, en les mettant en scène de façon très visible, ce qui n'était pas la pratique habituelle. Comme les commanditaires sont mêlés au reste du public, en donnant à voir au corps de métier mis à l'honneur sa propre image (laudatrice et valorisée), on la donne à voir aussi au reste de la population, de façon d'autant plus efficace que la représentation de ces pièces était reprise à divers endroits de la ville de Badajoz, et non pas limitée au public de la bourgade de Talavera ${ }^{43}$. La façon dont sont évoquées les professions renforce non seulement la théâtralité de ces

41 " que amo tengo que los coma ", v. 140.

42 On trouve toutefois un bref développement de la description de l'activité des ouvriers agricoles travaillant dans une vigne dans la Representación de la parábola de San Mateo, de Sebastián de Horozco. Voir S. de Horozco, Representaciones, F. González ollé, Madrid, Castalia, 1979, p. 67-97.

43 La destination de ces pièces à un public ouvert explique certainement le paradoxe, dans la Farce du Meunier, de la présence conjointe d'éloge et de satire (indulgente, toutefois), le public de la rue s'attendant nécessairement à voir apparaître sur scène les éléments que la tradition populaire attribue à ce personnage. 
pièces, mais aussi leur fonction célébrative : les commanditaires ont l'occasion de se réjouir de la fête du Saint-Sacrement en tant que membres de la communauté chrétienne, mais aussi en tant que personnes ayant concouru à donner tout son éclat à cet événement festif.

$\mathrm{Au}$ théâtre, on l'a vu à travers les exemples cités, le travail peut apparâ̂tre sous la forme d'une représentation directe de la profession ou sous forme de récit et de description faits sur scène par un personnage. Le prologue de ces farsas, est, par nature narratif et descriptif, ce qui explique que l'essentiel du portrait des professions se situe dans cette partie des pièces. C'est au contraire dans le corps de la pièce que figure généralement l'exploitation symbolique de ce métier sur le plan religieux, et dans les parties comiques qu'est mise en scène la profession par des actions, comme c'est le cas de deux autres professions qui n'ont pas été encore mentionnées ici, celle du Maestro de sacar muelas ${ }^{44}$ (' l'arracheur de dents ') et du Potrero ${ }^{45}$ (' celui qui opère les hernies '). Ces deux professions font leur apparition dans une partie comique finale de leurs pièces respectives, en tant qu'éléments de l'action. Dans les deux cas, il s'agit de punir un personnage malhonnête et ridicule, et ces personnages sont, en quelque sorte, les exécutants de la justice dramatique. Ces professions sont mises en théâtre pour les nécessités de l'action de la pièce plutôt que pour elles-mêmes : elles ne sont pas l'objet de satire, ni d'éloge, ni d'exploitation symbolique. Ces deux contre-exemples montrent que, dans ce thêâtre, le véritable discours valorisant sur le travail et les métiers est tenu de façon strictement verbale plutôt que sous forme d'action, ce qui n'est pas étonnant dans ce que l'on considère comme un " théâtre du verbe ". 